ARTIGO ORIGINAL ORIGINAL ARTICLE

\section{Registo de saúde eletrónico: contributos para novos modelos organizacionais no sector público da saúde}

\author{
Electronic health record: contributions to new \\ organizational models in the public health sector \\ Pedro Miguel Alves Ribeiro Correia', Helena de Jesus Fernandes Ruivo Bernardes
}

DOI: 10.21115/JBES.v9.n2.p185-97

\section{Palavras-chave:}

administração pública, administração de saúde, modelos de gestão, registo de saúde eletrónico, enfermagem

\section{Keywords:}

public administration, health administration, management models, electronic health record, nursing

\section{RESUMO}

Objetivo: O objetivo deste trabalho foi analisar os Registos de Saúde Eletrónicos, pertencente a utentes com internamento, numa unidade de cuidados de um hospital público, em Portugal. Métodos: Foram utilizados dados relativos ao ano de 2014 e 2015, referentes a 668 doentes, com 14.001 dias de internamento, 4.123 diagnósticos e 614.634 intervenções, pelo que podemos considerar a análise empírica robusta. Resultados: Em ambos os anos, é notório que os dados são bem aproximados por funções exponenciais, o que fica espelhado pelos coeficientes de determinação extremamente elevados, obtidos para cada um dos casos. Este facto têm como implicação que a maior parte do trabalho efetivo dos enfermeiros desta unidade de cuidados advém de um pequeno número de tipos de intervenção, sendo possível argumentar, de forma análoga ao verificado para os diagnósticos, o que tem impactos importantes ao nível da gestão. Conclusões: A análise dos dados revelaram potencial para a criação de indicadores e análise da qualidade. Os Registos de Saúde Eletrónicos permitem a medição de resultados e também a identificação de melhores práticas clínicas associadas a esses resultados, devendo ser visto como uma ferramenta de gestão e de investigação, que pode apoiar processos de trabalho e de inovação na prestação de cuidados. 


\section{Introdução}

A rápida evolução do conhecimento e da tecnologia, condiciona a sociedade e a vida do Homem, aos mais diferentes níveis. A crescente evolução das Tecnologias de Informação e Comunicação têm promovido o aparecimento nas organizações de diferentes estratégias e ferramentas, incluindo as utilizadas na gestão. Esta nova era da informação trouxe mais dados, maior complexidade e maior capacidade de monitorização, o que implica uma reorganização dos métodos de trabalho e a uma reformulação das metodologias na gestão. Para atingir objetivos e metas, cada organização utiliza estratégias de gestão aproveitando todos os recursos disponíveis, nomeadamente os relacionados com as Tecnologias de Informação e Comunicação.

No sector público, a gestão e os modelos organizacionais sofreram uma evolução, e mudanças importantes. Embora na atualidade se encontrem diferentes modelos de gestão, nas nossas organizações, o New Public Management trouxe novas formas de gestão, promoveu novas técnicas e padrões de gestão pública, por forma às organizações se tornarem mais efetivas e eficientes (Simione, 2014). Harfouche (2016) considera que a New Public Management introduziu métodos oriundos do privado na área pública, promovendo uma gestão centrada no aumento da eficácia e eficiência, e introduzindo mecanismos de avaliação de performance, como a criação de indicadores. Neste âmbito, Osbone et al. (2013) referem que o desenvolvimento das Tecnologias de Informação e Comunicação colocaram novos desafios para a gestão de serviços. Com modelos de gestão no sector público cada vez mais semelhantes ao do sector privado, onde se utilizam métodos como o planeamento e monitorização de indicadores e Objetivos, as Tecnologias de Informação e Comunicação assumem cada vez mais um papel importante.

A área da saúde é uma grande produtora de informação e alvo de grandes transformações. O panorama da prestação de cuidados de saúde está em transformação, com o desenvolvimento de conceitos e ferramentas necessárias à implementação de um verdadeiro modelo orientado para os resultados em saúde mais relevantes (Boston Consulting Group [BCG], 2016). Alguns autores (Villax (Ed.), 2015) acrescentam que a sustentabilidade da saúde têm como elementos centrais a inovação tecnológica e as iniciativas baseadas no conhecimento. É um facto que os cuidados de saúde são uma atividade que implica bastante informação, pelo que existem ganhos potenciais na implementação de novas tecnologias como a eletrónica e o eHealth, que podem também modificar e tornar mais eficiente a gestão (Carrasqueiro \& Monteiro, 2010).

O termo eHealth é definido como "(...) a aplicação das tecnologias da informação e das comunicações em toda a gama de funções que afetam o sector da saúde." (Organization for
Economic Cooperation and Development/European Union [OECD/ EU], 2016, p. 178). Os registos de dados, realizados pelos profissionais de saúde, no processo clínico do utente alvo de cuidados, ou Registos de Saúde Eletrónicos, está incluído nas diferentes soluções eHealth, e são para Carrasqueiro e Monteiro (2010) "(...) serviços baseados em sistemas de informação que implementam meios para registrar, recuperar e compartilhar informações clínicas" (p. 462). Trata-se de um recurso importante no sector da saúde, de uma ferramenta de grande potencial no apoio à decisão, aos mais diferentes níveis, da prática clínica à gestão. Das diferentes capacidades deste Registo de Saúde Eletrónico Odekunle (2016) refere ainda a visão integrada de informações e dados sobre o utente do sistema de saúde, mas também o acesso a recursos valiosos de conhecimento. A aplicação ampla e adequada destes Registos de Saúde Eletrónicos no sistema de cuidados de saúde têm ainda um grande potencial no apoio aos profissionais de saúde, ao reduzir os erros médicos, a conseguir uma meIhor coordenação e eficácia dos cuidados, ao melhorar a segurança e a qualidade dos cuidados, e ainda ao reduzir os custos na saúde (Odekunle, 2016). As instituições destinadas aos cuidados de saúde, à semelhança de outras entidades empresariais, são empresas que utilizam muita informação, pelo que é importante que os profissionais de saúde utilizem dados, e ferramentas adequadas, para fazer uma boa gestão da informação, de forma a tomar decisões precisas aos mais diferentes níveis (Odekunle, 2016).

Neste trabalho é dada especial atenção a um grupo profissional da área da saúde com grande expressão em número e volume de cuidados, os enfermeiros (Instituto Nacional de Estatística [INE], 2016), com implicações importantes na qualidade e sustentabilidade do sistema de saúde. O foco de atenção dos autores foram os Registos de Saúde Eletrónicos de uma importante instituição de saúde do sector público, do centro do país. Ao analisar o conteúdo da informação, produzida ao longo de dois anos (2014 e 2015) pelos enfermeiros, procuram responder às seguintes questões:

1. Que evidências existem nos dados do Registo de Saúde Eletrónico, produzidos pelos enfermeiros de uma unidade de cuidados hospitalares do sector público?

2. Quais os contributos do Registo de Saúde Eletrónico para a caracterização da qualidade e produtividade dos cuidados de enfermagem de uma unidade de cuidados hospitalares?

Assim, o objetivo geral deste trabalho foi analisar os Registos de Saúde Eletrónicos, pertencente aos utentes, com episódio de internamento numa unidade de cuidados de um hospital público em Portugal, no período de 2014 e 2015. Como Objetivos específicos definimos: Reunir os principais dados padronizados de todos os utentes com episódios de internamento e alta durante os anos de 2014 e 2015; Conhe- 
cer e descrever os padrões de diagnósticos e intervenções de enfermagem; Conhecer e descrever os indicadores que mais se destacaram nos dados; Conhecer e descrever as áreas com necessidade de plano de melhoria da qualidade. Esta análise foi enquadrada com diferentes teorias sobre Administração Pública, Gestão e Inovação, como forma de contextualização.

\section{Enquadramento}

Os cuidados de saúde envolvem um grande volume de complexa informação, e a evolução das Tecnologias de Informação e Comunicação não só elevaram as expectativas, mas também as exigências aos mais diferentes níveis neste sector. Estas tecnologias evoluíram em diferentes áreas e transformaram antigos paradigmas nas organizações. O sector da saúde, embora tradicionalmente mais conservador, também se debate com novos desafios nas organizações. A gestão assume um papel primordial, na adoção de novas estratégias, na implementação de novos projetos tecnológicos e na reorganização do trabalho. Silva (2004) refere que "O modelo organizacional é um meio facilitador da inserção de tecnologias de gestão, produção e de comunicação, mesmo sendo tecnologias com origem noutras indústrias." (p. 24). Este autor, considera que as organizações devem adaptar-se permanentemente à evolução da sociedade, e criar condições face às alterações estruturais que o crescente conhecimento tecnológico impõe. Para Druke e Klinger (2011) também a Administração Pública sofre com a pressão da mudança. Para estes autores, a utilização destas novas tecnologias são importantes elementos impulsionadores da inovação e modernização do sector público.

A gestão numa organização utiliza diferentes ferramentas, sendo a informação uma poderosa aliada em muitos âmbitos, incluindo na criação de indicadores, sua monitorização e avaliação. Silva (2004) considera que uma unidade organizacional deve construir indicadores e avaliar os resultados dos seus processos, identificando as competências necessárias, captando tendências requeridas no futuro, de forma a dar uma resposta eficiente e eficaz. Esta abordagem, centrada na eficiência, qualidade e excelência, com gestores que procuram acompanhar e medir resultados dos diferentes processos, enquadra-se num modelo a que Silva (2004) denomina de gestão gestionária. No sector público este é um grande desafio, e é para Simione (2014) uma necessidade premente, de forma a se constituir um sector cada vez mais capacitado. Capacitado para poder responder às solicitações da sociedade, para uma prestação de serviços públicos com maior qualidade, assim como, para uma elevação do desempenho, o que realça mais uma vez o papel primordial da gestão.

Já as conceções da New Public Management, surgidas na década de 80 , sugeriam novas formas de gestão, com novas estratégias, técnicas e padrões para o sector público, por for- ma a transformar as organizações em entidades mais efetivas (Simione, 2014). Secchi (2009) aborda a temática da gestão e dos modelos organizacionais, bem como a forma como os quatro principais modelos inspiraram as reformas da Administração Pública. Este autor considera que no sector público ainda subsiste a evidência de um convívio de diferentes modelos organizacionais, em especial os quatro seguintes: o Burocrático, a Administração Pública Gerencial, o Governo Empreendedor e a Governança Pública.

O modelo Burocrático ou Weberiano é descrito por Secchi (2009) como ultrapassado, ineficaz, centrado numa visão normativa e legal, e afastado dos cidadãos. Mas, este modelo cujas algumas das características ainda persistem nas organizações, teve um apogeu e fez parte de reformas importantes na Administração Pública, ficando conhecido como Progressive Public Manager (Secchi, 2009). Após a segunda guerra mundial surgiram os primeiros sinais de necessidade de mudança, e três novos modelos conhecidos por: a Administração Pública Gerencial e o Governo Empreendedor (fazendo parte do Gerencialismo) e a Governança Pública. Na Administração Pública Gerencial, modelo também conhecido por New Public Management, os principais valores que lideraram a mudança foram: a eficácia, a eficiência e a competitividade (Secchi, 2009). A eficiência é um valor partilhado por um outro modelo, o Governo Empreendedor (Secchi, 2009). Para este autor, estes dois modelos partilham ainda outras características, que descreve como: a produtividade, a orientação para o serviço, a descentralização, a utilização de ferramentas de mercado (Marketization) e ainda a Accountability (responsabilidade, transparência, controle). A Governança Pública é considerado o modelo de modernização das organizações públicas, inspirado nos conhecimentos do sector privado, que associa uma mudança do papel do Estado (Secchi, 2009).

Bilhim (2004) considera que uma nova visão da Administração Pública conhecida por "novo serviço público" (p.30) apresenta um conjunto de lições práticas, que desafiam as práticas tradicionais, e que requer novos modelos de gestão. De facto, o New Public Management introduziu métodos oriundos do privado na área pública, promovendo uma gestão centrada no aumento da eficácia e eficiência, introduzindo mecanismos de avaliação de performance como a criação de indicadores e sua monitorização (Harfouche, 2016). Para este autor, as necessidades de reforma da Administração Pública, numa tentativa de modernização da sua estrutura, tiveram reflexo em especial na área da saúde. Estas mudanças permitiram alterações significativas e mudanças entre os antigos modelos tradicionais até ao New Public Management, e mais recentemente, o New Public Service, baseado no interesse publico, centrado no cidadão e na cidadania como prática estruturante (Harfouche, 2016). A intervenção do Estado passou para um lugar de menor destaque, os modelos de gestão das unidades de saúde foram sendo alterados, des- 
tacando-se estratégias do sector privado, como a procura da eficiência e a monitorização de indicadores (Harfouche, 2016).

Os cuidados de saúde constituem uma área crítica em relação a todas estas questões. A este propósito Taylor et al. (2015) analisaram fatores associados, e estratégias práticas, de melhoria da performance dos hospitais, e da sua eficiência. Neste trabalho, estes autores identificam sete áreas que representam fatores associados ao alto desempenho, salientando a monitorização efetiva do desempenho. O tema da monitorização é para estes autores apoiado por sistemas de dados sofisticados, sendo benéfico investir recursos e infraestruturas que funcionem por forma a monitorizar o desempenho clínico e financeiro, e assim apoiar a melhoria contínua da qualidade. A transformação do sector da saúde deve estar centrada nos resultados (BCG, 2016). Para tal, é importante adotar indicadores de resultados em saúde, e é imprescindível criar um sistema de registos que reúna uma certa qualidade de dados, referentes aos cuidados de saúde (BCG, 2016). Gestão de cuidados de saúde baseada na monitorização dos resultados privilegia a inovação e o foco na obtenção de ganhos em saúde, criando ao mesmo tempo uma ênfase no valor e na sustentabilidade (BCG, 2016).

Como já foi referido, os enfermeiros constituem o grupo profissional de maior dimensão no sector da saúde (INE, 2016) pelo que são responsáveis por um grande volume de cuidados de saúde e também de informação. A Ordem dos Enfermeiros, preocupada com a questão da qualidade dos cuidados prestados, publicou Padrões de Qualidade com enunciados descritivos, com o objetivo de melhorar os cuidados a fornecer aos cidadãos (Ordem dos Enfermeiros [OE], 2001). Para esta Ordem profissional "Criar sistemas de qualidade em saúde revela-se uma ação prioritária" (OE, 2001, p. 5-6). Estes padrões foram conceptualmente enquadrados, promovendo a implementação de projetos no âmbito da qualidade dos cuidados, em diferentes instituições de saúde, monitorizados através de indicadores, evidenciando as novas tendências da gestão no sector da saúde, que coloca um particular destaque na eficiência e eficácia.

De facto, o sector da saúde envolve um esforço intensivo em informação, nas novas tecnologias, originando novas terminologias neste cenário. A adoção de tecnologia digital na saúde, também denominado eHealth, pode promover e apoiar a melhoria dos cuidados (OCDE/ EU, 2016). Organizações internacionais consideram que estes benefícios incluem também a melhoria no planeamento e na gestão (OECD/ EU, 2016). A informação em cuidados de saúde assume um grande protagonismo na figura do Registo de Saúde Eletrónico, do utente do sistema de saúde, pois constitui a evidência do estado e dos cuidados de saúde à pessoa, podendo contribuir para a monitorização de diferentes indicadores, bem como, para a análise do desempenho do sistema. Monteiro (2010) apresenta dezasseis grandes grupos denominados serviços de eHealth, entre os quais se enquadra o Registo de Saúde Eletrónico que define como: "Electronic Health Record (EHR) and Online Clinical Transactions - These are services based on information systems which implement means to register, retrieve and share clinical information (...)" (p. 106). Kierkegaard et al. (2014) referem que ainda permanecem desafios no aumento do uso desta informação registada ao longo dos cuidados de saúde. Estes autores consideram que os dados podem fornecer padrões de cuidados aos utentes e fornecer dados que podem melhorar a gestão dos cuidados, contribuindo para uma potencial economia e equilíbrio dos gastos na saúde. Um melhor uso dos dados e da tecnologia têm o poder de melhorar a qualidade dos cuidados de saúde e de reduzir o custo dos serviços e dos cuidados (National Information Board, 2014).

As Tecnologias de Informação e Comunicação aplicadas à saúde podem aumentar a eficiência e a qualidade, princípios importantes nos novos modelos de gestão, e podem ainda estimular a inovação nos mercados da saúde (Comissão Europeia, 2012). Mas, as diferentes dimensões de saúde, que a Organização Mundial de Saúde preconiza, devem estar refletidas no Registo de Saúde Eletrónico (Organização Mundial de Saúde [OMS], 2016). Villax (2015) chama a atenção para outro aspeto importante do Registo de Saúde Eletrónico, o de permitir o apoio à investigação. Este autor dá também um outro destaque ao seu potencial da seguinte forma: "Melhorar a saúde, os cuidados de saúde e o bem estar dos utentes através da gestão dos seus registos de saúde (...)" (Villax, 2015, p. 11). De facto, a proliferação da tecnologia transformou a forma como a informação é capturada, processada, armazenada e analisada. O termo eHealth é definido como "a aplicação das tecnologias da informação e das comunicações em toda a gama de funções que afetam o sector da saúde" (OECD/ EU, 2016, p. 178). Para Carrasqueiro e Monteiro (2010) a OMS define eHealth como:"(...) a utilização combinada no sector da saúde das comunicações eletrónicas e das tecnologias da informação (...) para fins clínicos, educativos e administrativos (...)"(p. 452).

Segundo a Administração Central do Sistema de Saúde (Portugal, 2009) o Registo de Saúde Eletrónico deveria cumprir os desígnios de partilha de informação de saúde, centrada no utente, e orientada para o apoio ao cumprimento da missão dos profissionais de saúde, princípios presentes nos novos modelos organizacionais. A disponibilização de um sistema de registo, alicerçado na utilização de ferramentas indutoras de eficácia e eficiência da prestação de cuidados de saúde, traz ainda benefícios acrescidos, possibilitando"(. . .) Melhorar de forma significativa os indicadores de gestão (...)" (Portugal, 2009, p.6).

Parece claro, para diferentes autores, que o Registo de Saúde Eletrónico permitirá a medição de resultados, e a identificação das melhores práticas clínicas associadas a esses resultados. A grande revolução consiste na utilização destas 
novas tecnologias numa combinação, com uma mudança nos modelos de organização e gestão nos sistemas de saúde, por forma a melhorar os cuidados de saúde, não esquecendo a eficiência, a produtividade, o valor económico e social de saúde (BCG, 2016). O Registo de Saúde Eletrónico deve ser visto como uma ferramenta de gestão e de investigação, que pode apoiar processos de trabalho e de inovação na prestação de cuidados (Poissant et al., 2005). A utilização destes registos permite entender padrões de práticas e cuidados, bem como a eficiente utilização dos recursos (Odekunle, 2016). Odekunle (2016) refere que têm sido demonstrado que o Registo de Saúde Eletrónico desempenha um papel relevante, e sua aplicação abrange muitos aspetos do sistema de saúde. Os principais fatores para o papel crescente destes registos incluem, a necessidade de melhorar a eficiência na prestação de serviços, a segurança do utente, aumentar o acesso e a necessidade de reduzir os custos (Odekunle, 2016).

Uma eficaz utilização do Registos de Saúde Eletrónico com todos estes objetivos requer uma padronização da linguagem, o que têm preocupado diferentes profissionais do sector. Os enfermeiros tomam decisões em grande volume, muitas delas complexas, reunindo assim muita informação (Simões \& Simões, 2007). Aposta-se cada vez mais, para estes profissionais, em sistemas de registo que permitam a utilização da informação recolhida para as diferentes finalidades (Simões \& Simões, 2007). Neste âmbito, as organizações profissionais preocuparam-se com a padronização dos registos de forma a promover e facilitar a criação de indicadores, a sua monitorização e a comparação de resultados. A profissão de Enfermagem é uma profissão e disciplina científica, sendo que a consolidação e manutenção do seu corpo de saber requer uma permanente atualização, fundamentada cientificamente em dados, que a informatização pode sem dúvida alguma melhorar (Simões \& Simões, 2007). Para a Ordem dos Enfermeiros, e para o Conselho Internacional de Enfermeiros (2016), é um facto incontestável que "Enfermeiros em todos os países e em todos os contextos de prática originam, recebem e gerem uma enorme quantidade de informações diariamente. A disponibilidade de boa informação é um pré-requisito para cuidados eficazes de alta qualidade." (p. 13).

É neste contexto que nasce a Classificação Internacional para a Prática de Enfermagem (CIPE). A CIPE é uma classificação, promovida pelo International Councel of Nurses, que têm vindo a evoluir nos últimos 25 anos, encontrando-se implementado em 18 idiomas, em diferentes países (Ordem dos Enfermeiros [OE] \& Conselho Internacional de Enfermagem [CIE], 2016). Trata-se de uma forma padronizada de registar informação sobre os cuidados de saúde prestados pelos enfermeiros, que utiliza uma linguagem baseada e categorizada em diagnósticos (problemas do utente alvo dos cuidados de enfermagem) e intervenções de enfermagem (OE \& CIE, 2016). Para que a enfermagem contribua para os sistemas de informação multidisciplinares, o International Councel of Nurses trabalhou em colaboração com a Organização para o desenvolvimento de Normas Internacionais de Terminologia da Saúde (OE \& CIE, 2016). Com dados registados de forma padronizada, e linguagem codificada, pode gerar-se muita informação e indicadores, que permitem monitorizar e analisar os cuidados, os recursos e os resultados dos cuidados, numa perspetiva centrada na pessoa (OE \& CIE, 2016).

Baseada nesta padronização CIPE, e com este novo foco de atenção de uma gestão centrada em objetivos e indicadores, a Ordem dos Enfermeiros publicou um documento com um quadro de referência, para a construção de indicadores de qualidade e produtividade em Enfermagem (Ordem dos Enfermeiros [OE], 2007). O objetivo foi definir as linhas gerais de um modelo, dos seus conteúdos e dos requisitos de comparabilidade, para um Resumo Mínimo de Dados de Enfermagem, que viabilizem a produção de um conjunto de indicadores de cuidados de enfermagem, para diferentes níveis e utilizadores, mas que merece um foco de atenção especial para os gestores. O documento determina indicadores de estrutura, de processo, de resultados e epidemiológicos, que são descritos da seguinte forma: taxa de efetividade diagnóstica do risco, taxa de efetividade na prevenção de complicações, Modificações positivas no estado dos diagnósticos, taxas de ganhos possíveis / esperados de efetividade, taxas de incidência, taxas de prevalência e taxas de frequência relativa $(O E, 2007)$. A criação destes indicadores teve em atenção a mensurabilidade através de dados diários do Registo de Saúde Eletrónico, e foram enquadrados nos padrões de qualidade enunciados pela Ordem dos Enfermeiros (OE, 2007).

As mudanças nos modelos organizacionais da Administração Pública assumem uma expressão particular no sector da saúde, como foi apresentado nesta breve contextualização. Num sector onde se evidência um convívio de diferentes modelos organizacionais, sobressaem estratégias do New Public Management e das suas ferramentas oriundas do privado, de mensurabilidade e promoção da eficácia e da eficiência. Também os profissionais de saúde, nomeadamente os enfermeiros, são alvo destas transformações, ao adotarem ferramentas inovadoras que permitem medir e monitorizar.

\section{Métodos}

Para o presente trabalho foram utilizados dados dos Registos de Saúde Eletrónicos de uma unidade de cuidados com internamento, de um importante hospital público, da zona centro do país. Um hospital é um estabelecimento de saúde dotado de serviços diferenciados, de capacidade de internamento, de resposta em regime ambulatório e de meios de diagnóstico e terapêutica, com o objetivo de prestar ao cidadão cuidados de saúde ao nível curativo, de reabilitação e de prevenção da doença (Ciências da Informação e da 
Decisão em Saúde [CIDES], 2011). Os dados em análise foram produzidos num hospital que também assume uma missão no ensino, sendo assim também uma escola de formação de diferentes profissionais de saúde.

A unidade de cuidados a que nos referimos é considerada de grande diferenciação técnica ao nível dos cuidados, e dá resposta a utentes com doença do foro infecioso, sob forma de internamento, mas também em regime ambulatório (consulta e hospital de dia). As doenças infeciosas são responsáveis por cerca de 19,2\% dos casos de morbilidade a nível mundial, e por cerca de 10 milhões de mortes (World Health Organization [WHO], 2015). A unidade de cuidados a que nos referimos destina-se à prestação de cuidados de saúde à pessoa com este tipo de patologias infeciosas, que podem ser muito variadas, desde infeções que envolvem qualquer órgão ou sistema, às infeção pelo Vírus da Imunodeficiência Humana, à sida ou de outras etimologias infeciosas. Têm capacidade assistencial para 25 doentes em camas (23 camas mais duas suplementares), e conta com uma equipa multidisciplinar da qual fazem parte um total de 18 enfermeiros, número que inclui o enfermeiro em funções de gestão. Trata-se de uma equipa com um tempo médio de experiência profissional na área da infeciologia de cerca 5 anos, o que revela um nível de competência elevado (Serrano et al., 2011). De uma forma global, o desempenho assistencial desta unidade de cuidados, nos anos em destaque no estudo (2014 e 2015), evidenciou um aumento da atividade, embora se tivesse registado uma diminuição geral na instituição de que falamos. Existem diferentes aplicações informáticas, fornecidas pelo Ministério da Saúde e Administração Central do Sistema de Saúde, sendo que a equipa de enfermagem utilizou, durante o período a que se refere o estudo, a aplicação informática GlinttHS, com o seu desktop de enfermagem, de onde foram retirados os dados. Os objetivos da assistência fornecida por esta equipa enquadra-se com os objetivos e princípios da instituição, e caracterizam-se da seguinte forma: Garantir cuidados de enfermagem de excelência; Constituir uma referência para a instituição, para a profissão, para os utentes e familiares e também na área da formação. A organização dos cuidados de enfermagem nesta unidade têm subjacente o método de responsável global pela prestação de cuidados ao utente e família, desde o acolhimento à alta.

A análise dos dados aqui apresentada encontra-se não só subdividida por ano, mas também por duas categorias de análise: frequência absoluta de cada tipo de diagnóstico de enfermagem e frequência absoluta de cada tipo de intervenção de enfermagem.

Em 2014 este serviço recebeu um total de 377 doentes, dos quais 354 obtiveram alta e 23 faleceram (taxa de mortalidade de 5,23\%). O total de dias de internamento foi de 6.631 dias, com uma média correspondente a 17,6 dias. Em 2014, estavam disponíveis no sistema informático 61 tipos de diagnósticos de enfermagem e 160 tipos de intervenções distintas. Foram efetuados um total de 2.027 diagnósticos aos quais corresponderam 274.251 intervenções.

Por seu turno, em 2015 este serviço recebeu um total de 291 doentes, dos quais 281 obtiveram alta e 10 faleceram (taxa de mortalidade de 2,99\%). O total de dias de internamento foi de 7.370 dias, com uma média correspondente a 25,3 dias. Em 2015, estavam disponíveis no sistema informático 65 tipos de diagnósticos de enfermagem e 194 tipos de intervenções distintas. Foram efetuados um total de 2.096 diagnósticos aos quais corresponderam 340.383 intervenções.

Como tal, de forma agregada, são utilizados dados relativos a um total de 668 doentes, 14.001 dias de internamento, 4.123 diagnósticos e 614.634 intervenções. Por qualquer medida estatística utilizada, é possível, sem sombra de dúvida, considerar a análise empírica como extremamente robusta!.

\section{Resultados e discussão}

Os gráficos 1 e 2 apresentam, respetivamente, as frequências absolutas dos tipos de diagnóstico de enfermagem, para os anos de 2014 e 2015, realizados no serviço em análise (em escala logarítmica).

Em ambos os casos, é notório que os dados são bem aproximados por funções exponenciais, o que fica espelhado pelos coeficientes de determinação extremamente elevados, obtidos para cada um dos casos $\left(R^{2}=0,976\right.$ e $R^{2}=0,964$, respetivamente). Este facto tem como implicação que a maior parte dos diagnósticos advém de um pequeno número de tipos de condição, o que, é possível argumentar, poderá ter impactos importantes ao nível do formato de gestão implementado no serviço em causa.

Realce-se que, em 2014, dos 55 tipos de diagnósticos de enfermagem registados, 6 destes ${ }^{2}$ (correspondendo apenas a cerca de 10,9\% do total) contabilizaram 892 entradas (correspondendo a uns relevantes $44,0 \%$ do total).

De forma similar, em 2015, dos 60 tipos de diagnósticos de enfermagem registados, 6 destes $^{3}$ (correspondendo apenas a cerca de 10,0\% do total) contabilizaram 745 entradas (correspondendo a uns relevantes 35,5\% do total).

1 Por exemplo, caso se tratassem de amostras aleatórias, e considerando a fórmula de cálculo da dimensão amostral para proporções, estaríamos perante erros amostrais inferiores a 3,80\% para os utentes, $0,83 \%$ para os dias de internamento, $1,53 \%$ para os diagnósticos e 0,13\% para as intervenções. Não obstante, a recolha de dados foi censitária para qualquer um dos anos e categorias considerados, incluindo, virtualmente, todas as observações.

2 Cuidar da higiene pessoal; mobilidade; dor; cansaço; queda; hipertermia.

3 Hipertermia; cuidar da higiene pessoal; dor; mobilidade; queda; conforto. 


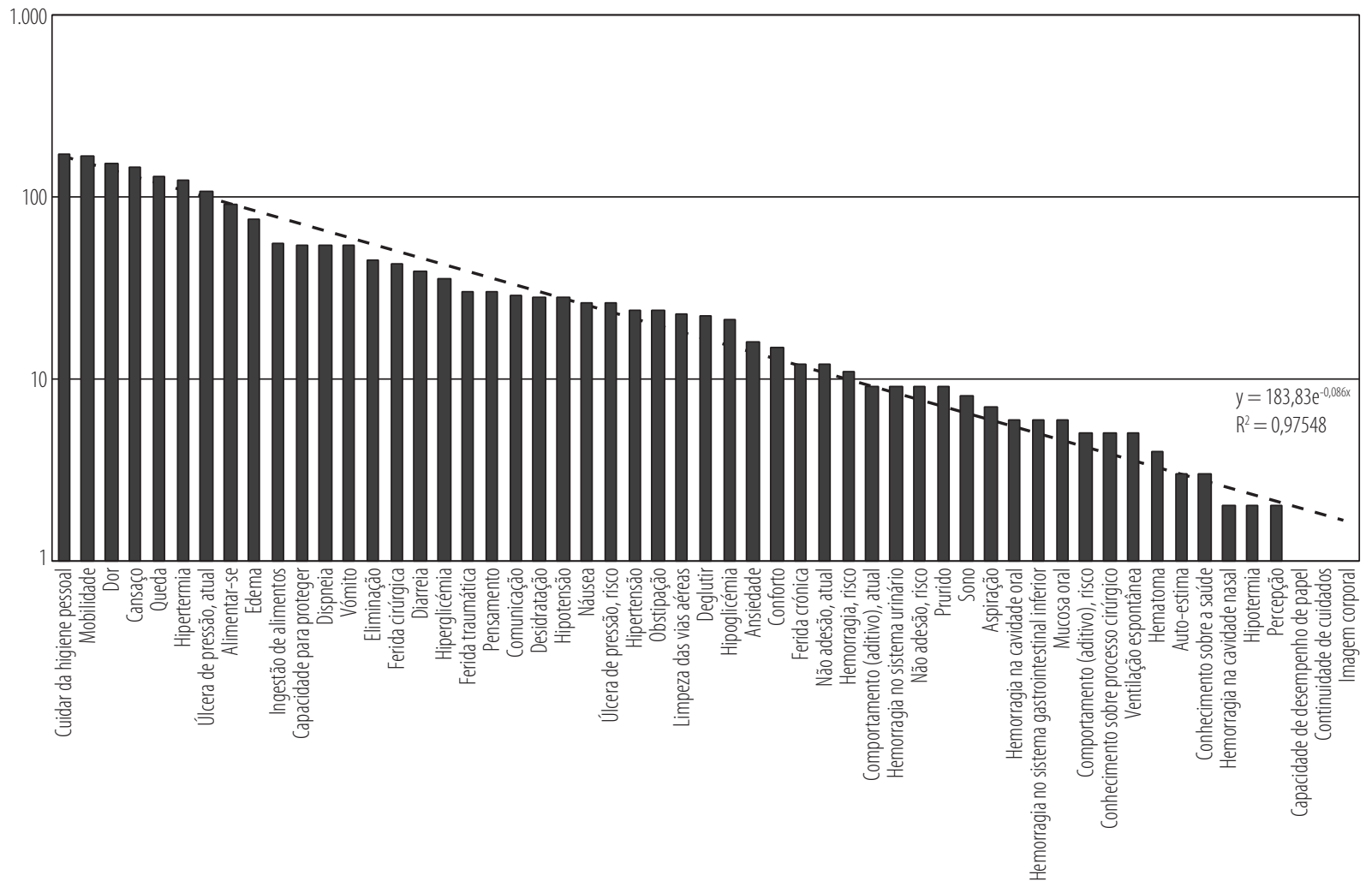

Fonte: elaboração própria com base em dados fornecidos pela instituição hospitalar em estudo.

Gráfico 1. Frequência absoluta dos tipos de diagnóstico de enfermagem, 2014 (escala logarítmica).

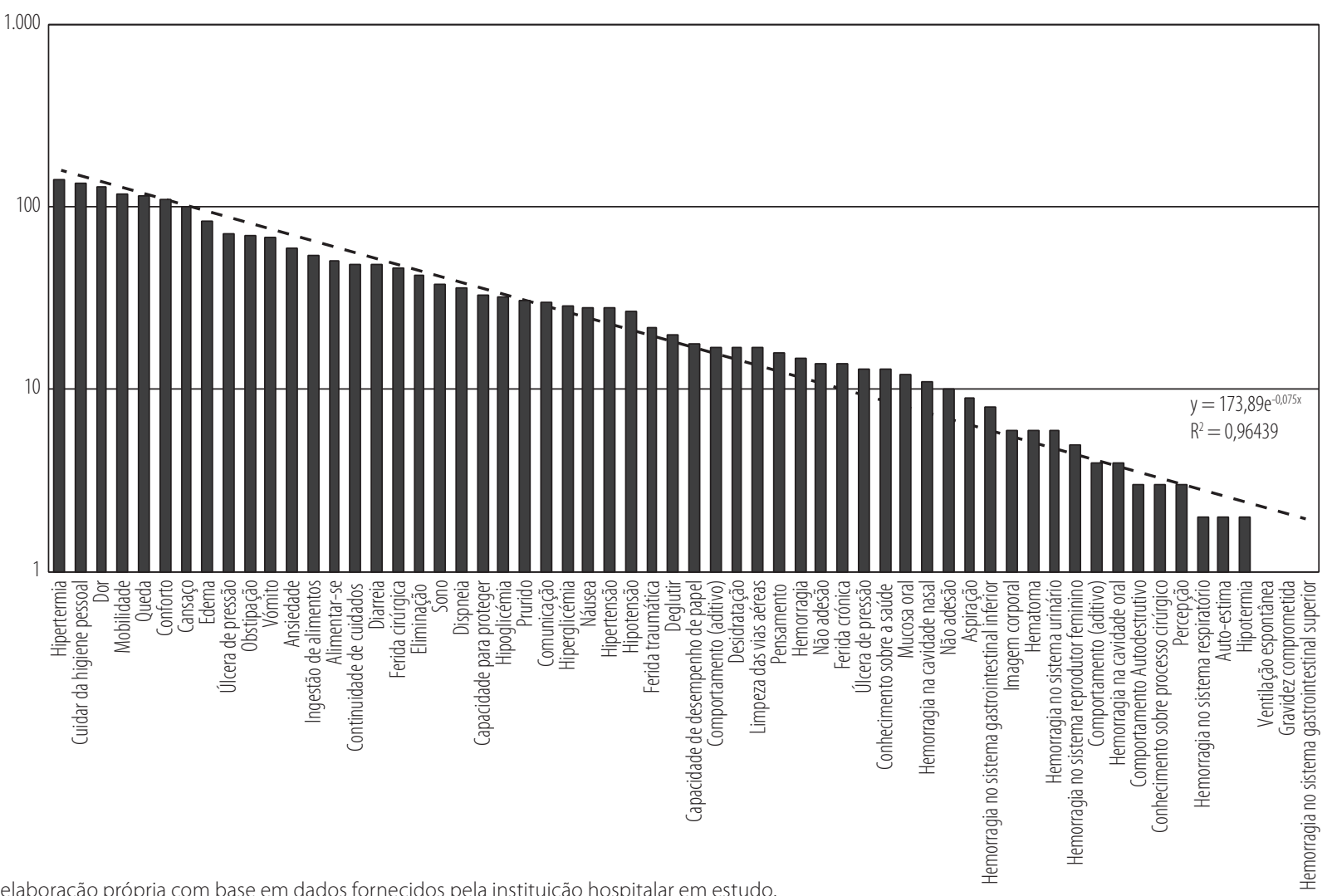


Os gráficos 3 e 4 apresentam, respetivamente, as frequências absolutas das intervenções de enfermagem, para os anos de 2014 e 2015, realizados na unidade de cuidados em estudo (em escala logarítmica).

Uma vez mais, em ambos os casos, é notório que os dados são bem aproximados por funções exponenciais, o que fica espelhado pelos coeficientes de determinação extremamente elevados, obtidos para cada um dos casos $\left(R^{2}=0,995\right.$ e $R^{2}=0,997$, respetivamente). Este facto têm como implicação que a maior parte do trabalho efetivo advém de um pequeno número de tipos de intervenção, o que, é possível argumentar, poderá ter, de forma análoga ao verificado para os diagnósticos, impactos importantes ao nível do formato de gestão implementado nesta unidade.

Realce-se que, em 2014, dos 118 tipos de intervenções de enfermagem registadas, 12 destas intervenções ${ }^{4}$ (correspondendo apenas a cerca de 10,2\% do total) contabilizaram 203.368 intervenções (correspondendo a uns expressivos $74,2 \%$ do total).

De forma similar, em 2015, dos 139 tipos de intervenções de enfermagem registadas, 14 destas intervenções ${ }^{5}$ (correspondendo apenas a cerca de 10,1\% do total) contabilizaram 226.587 intervenções (correspondendo a uns expressivos $66,6 \%$ do total)

Note-se que os fenómenos patentes nos gráficos 3 e 4 obedecem a leis exponenciais de forma consideravelmente mais intensa que os fenómenos patentes nos gráficos 1 e $2^{6}$.

Acrescentamos, que apesar de um decréscimo no número de utentes saídos no segundo ano (2015), estes apresentaram uma média de internamento aumentada, uma taxa de mortalidade mais reduzida, mas o número de diagnósticos de enfermagem aumentaram sensivelmente e, de forma mais marcada, as intervenções também. Estas evidências dos dados permitem-nos afirmar que no segundo ano (2015) o estado de saúde dos utentes agravou-se, com necessidades mais complexas ao nível dos cuidados, fundamentada por uma média de internamento aumentada, com uma maior

4 Vigiar ingestão de alimentos; monitorizar sinais vitais; vigiar pele; vigiar fezes; vigiar ingestão de líquidos; vigiar orientação; vigiar urina; executar técnica; posicionar o indivíduo; aplicar (protetor/tratamento cutâneo) em região corporal; monitorizar (glicemia capilar) do indivíduo; monitorizar (líquidos eliminados) pelo indivíduo.

5 Vigiar ingestão de alimentos; monitorizar sinais vitais; vigiar pele; vigiar fezes; vigiar orientação; executar técnica; vigiar ingestão de alimentos; vigiar ingestão de líquidos; vigiar mucosas; vigiar urina; posicionar o indivíduo; monitorizar (líquidos eliminados) pelo indivíduo; gerir (ambiente físico) do indivíduo; vigiar urina.

6 Fenómenos com comportamentos exponencias podem ser encontrados, de forma transversal, em vários setores da administração pública. Veja-se, como exemplo, Correia e Jesus (2016). necessidade de tempo de permanência de hospitalização, e também pelo aumento de diagnósticos e intervenções por parte da equipa de enfermagem. Por outro lado, estes dados revelaram também uma diminuição do resultado morte, evidenciado pela diminuição da taxa de mortalidade no segundo ano (2015) o que nos permite refletir sobre se houve uma melhoria dos cuidados globais aos utentes que originaram, ou contribuíram para melhores resultados.

Em ambos os anos deste estudo (2014 e 2015), e de forma global, a grande percentagem de levantamentos de diagnósticos de enfermagem (44\% e 35,5\% respetivamente) centraram-se em cerca de apenas 10\% do total de diagnósticos, e estes estavam relacionados com problemas de autonomia da pessoa (cuidar da higiene pessoal, mobilidade, conforto), mas também com questões de qualidade e segurança dos cuidados (dor, queda, hipertermia). Tratando-se de um hospital com internamento destinado ao tratamento da pessoa em situação aguda de doença, compreende-se esta centralidade de diagnósticos na autonomia, como problema foco de atenção das intervenções dos enfermeiros. Também os Padrões de Qualidade emanados pela Ordem dos Enfermeiros expressam essa preocupação, enquanto área de intervenção dos enfermeiros com potencial de produção de resultados positivos. A segurança é também um foco com uma expressão marcada nestes dados, e também faz parte dos Padrões de Qualidade. De salientar uma questão particular da qualidade, evidenciada pelo facto de que, apesar de um volume grande de problemas relacionados com a mobilidade e dependência de cuidados nas atividades diárias (cuidados de higiene e conforto), que pressupõe um maior risco de aparecimento de úlceras de pressão (risco associado à imobilidade), esse não têm expressão nos dados, o que nos leva a afirmar que a qualidade dos cuidados, e das intervenções desta equipa, neste campo, apresentam resultados muito positivos.

Também em ambos os anos (2014 e 2015) se verificaram um grande volume de registos (74,2\% e 66,6\%) em apenas cerca de 10\% das intervenções de enfermagem levantadas. Estas dizem respeito maioritariamente a intervenções de vigilância e monitorização de parâmetros fisiológicos da pessoa, que se justificam, em primeiro lugar pela vertente da profissão de despiste precoce de complicações na pessoa (Ordem dos Enfermeiros [OE], 2012), mas também pelo facto de muitas destas vigilâncias estarem relacionadas com rotinas diárias do utente (ingestão de alimentos implica varias observações no dia) ou rotinas das práticas diárias, com repetições em número elevado (monitorização de sinais vitais em todos os turnos). Também se evidenciaram intervenções com o objetivo de redução de riscos associados à imobilidade (diagnóstico frequentemente levantado), como gerir o ambiente físico do indivíduo, posicionar o indivíduo e aplicar (protetor/ tratamento cutâneo) em região corporal, o que decerto fez 

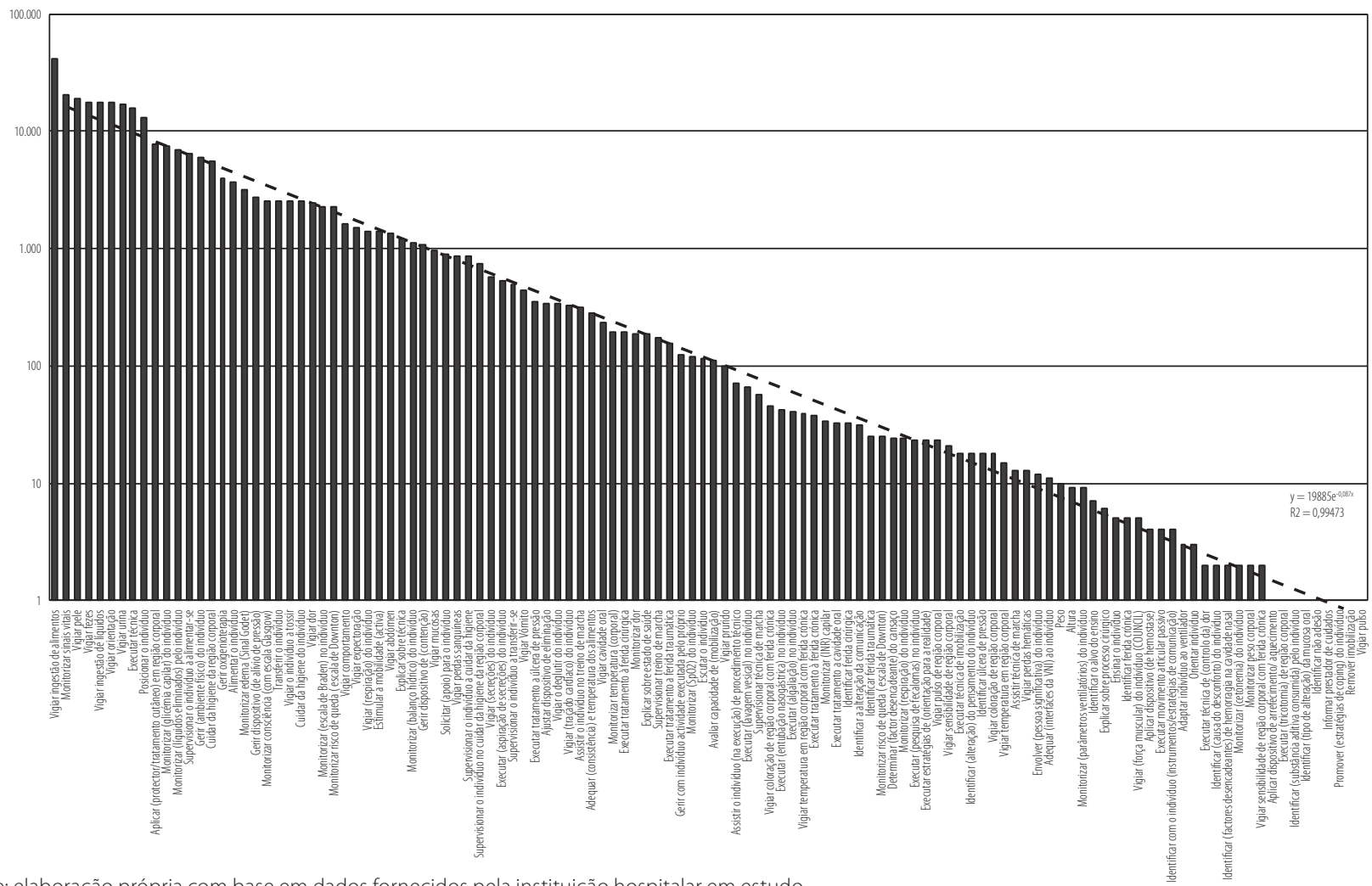

Fonte: elaboração própria com base em dados fornecidos pela instituição hospitalar em estudo.

Gráfico 3. Frequência absoluta das intervenções de enfermagem, 2014 (escala logarítmica).
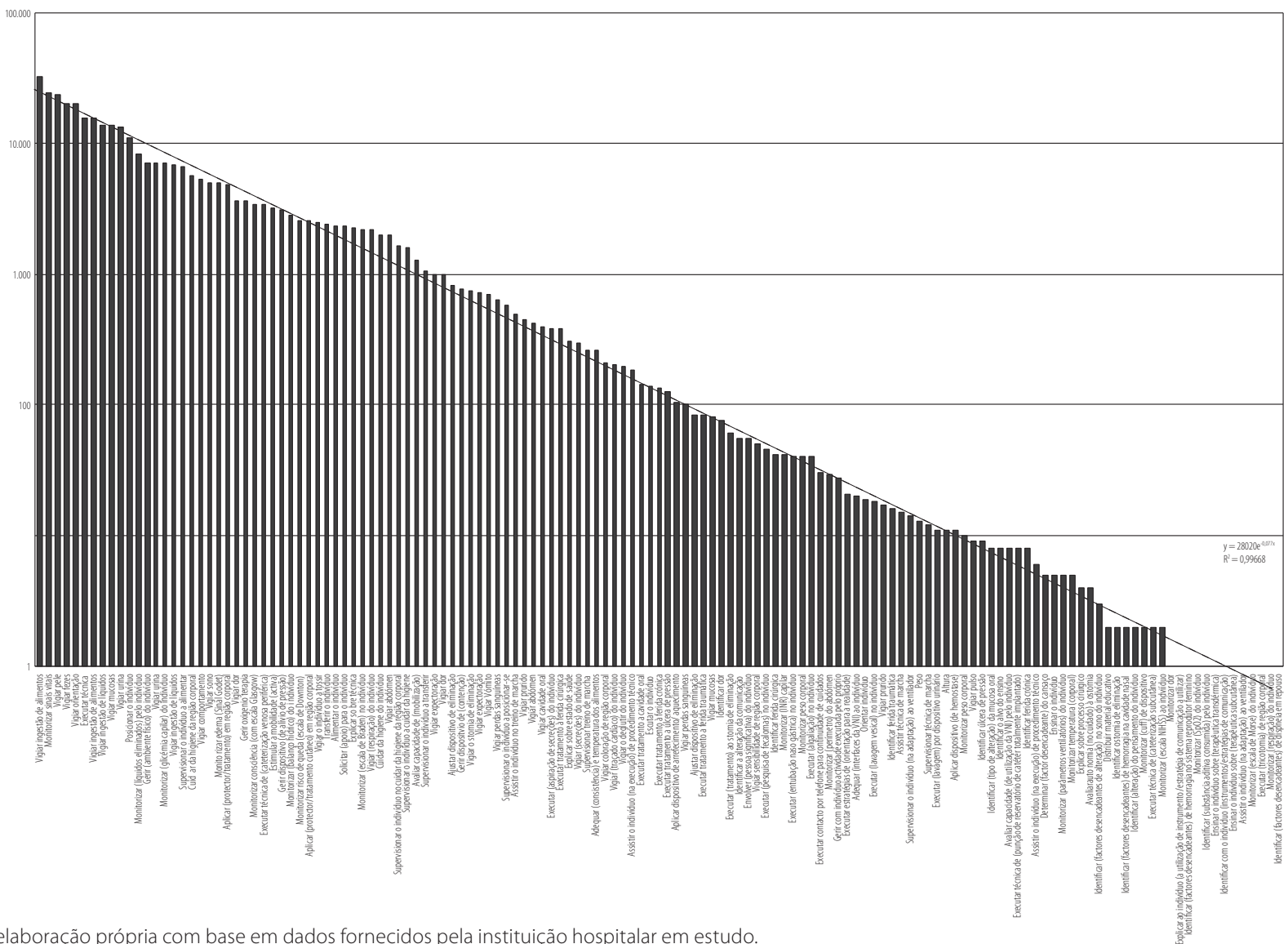

Fonte: elaboração própria com base em dados fornecidos pela instituição hospitalar em estudo.

Gráfico 4. Frequência absoluta das intervenções de enfermagem, 2015 (escala logarítmica). 
parte do sucesso à pouco referido, da pouca expressão de diagnósticos relacionados com a presença de úlceras de pressão, uma complicação associada com frequência a má qualidade dos cuidados. A intervenção "Executar Técnica" evidenciou-se, no entanto, esta pode estar relacionada com diferentes tarefas, tais como: colheita de espécimenes para análise, despiste de substância, cuidados a acesso venoso, entre outras. Tratando-se de uma profissão marcadamente técnica esta evidência não constituiu surpresa. Necessitaria, no entanto, de uma análise mais pormenorizada que estes dados fornecidos não permitiram.

O exercício da profissão de enfermagem implica um conjunto de competências, capacidades e conhecimentos, que podemos agrupar, por apresentarem características e padrões comuns, relacionadas com atividades e tarefas exercidas. Segundo a Ordem dos Enfermeiros (2012) "(...) o enfermeiro possui um conjunto de conhecimentos, capacidades e habilidades que mobiliza em contexto de prática clínica que the permitem ponderar as necessidades de saúde do grupo-alvo e atuar em todos os contextos de vida das pessoas, em todos os níveis de prevenção." (p. 7). Podemos afirmar, que as competências dos enfermeiros se agrupam em científicas, técnicas, relacionais e comunicacionais, sendo importante o desenvolvimento de todas estas ao longo dos cuidados (OE, 2012). Tendo em conta estes aspetos, e ao analisarmos os dados referentes a este estudo, podemos incluir os diagnósticos e intervenções de enfermagem que mais se evidenciaram em duas categorias, uma com componente marcadamente Instrumental, na gestão das atividades de vida diária do utente (alimentação, mobilização, eliminação, higiene), e outra com componente marcadamente Instrumental, na Gestão de Complicações (vigilâncias, monitorizações). No entanto, não se destacaram os diagnósticos e intervenções com componente marcadamente relacional e comunicacional, da qual fazem parte intervenções como as de promoção da saúde, ou de promoção de cuidados interprofissionais, ou ainda de referenciação de cuidados, o que levanta questões importantes numa profissão onde estas intervenções fazem parte do mandato, e cujas capacidades são primordiais e se encontram enquadradas e regulamentadas (OE, 2012). Segundo a Ordem dos Enfermeiros (2012) "O enfermeiro estabelece relações terapêuticas com o cliente e/ou cuidadores, através da utilização de comunicação apropriada e capacidades interpessoais." (p. 18). Na sua contribuição para a promoção para a saúde, conforme mandato social da profissão o enfermeiro "(. . ) dota os cidadãos de conhecimentos, capacidades, atitudes e valores que os ajudem a fazer opções e a tomar decisões adequadas ao seu projeto de saúde." (OE, 2012, p. 15). O enfermeiro também assume "(..) papel de interlocutor privilegiado da equipa pluriprofissional estando no centro dos cuidados com o cliente/ cuidadores, com estratégias de articulação assentes numa comunicação" (OE, 2012, p. 20).
A comunicação constitui uma ferramenta importante para todos os profissionais de saúde, pois é através dela que se estabelecem diagnósticos, planos de cuidados e promoção de ganhos em saúde para o cidadão. A propósito de estratégias comunicacionais nos cuidados de enfermagem Pontes et al. (2007) referem no seu artigo sobre registos de enfermagem que "(...) o relacionamento interpessoal e a comunicação não foram efetivos como deveriam ser, porém, não se pode negá-los nem fugir deles, em razão da sua importância para o processo de enfermagem." (p. 317). Estes autores referem ainda que "(...) precisa-se trabalhar e desenvolver estratégias de relacionamento interpessoal e com isso efetivar a comunicação entre enfermeiro e paciente, pois o processo de comunicação terapêutica deve ser priorizado como atividade de enfermagem relevante e essencial." (Pontes et al., 2007, p. 317).

Tratando-se esta questão como uma questão de qualidade, também outros autores se preocuparam em analisá-la com o foco nos registos, e na importância dada pelos enfermeiros às diferentes intervenções, competências e respetivos destaques dados na informação produzida. Silvestre (2012) refere que "É necessário que os enfermeiros documentem os cuidados que prestam, os diagnósticos, as intervenções e os resultados para que seja possivel evidenciar a qualidade" (p. 121). Também para este autor "(..) constatamos a necessidade de emergir novos estudos que identifiquem fatores determinantes para a não valorização de focos (...)" (Silvestre, 2012, p. 124).

A constatação da desvalorização dos enfermeiros de certos focos de atenção nos seus registos, pode dever-se a diferentes fatores, tais como "(...) equipa jovem pode levantar questões nomeadamente quanto à valorização dos registos, à aplicação da linguagem CIPE no dia-a-dia profissional, ao percurso académico e a formação em linguagem CIPE e (...) à aplicação, adaptação ao processo de mudança" (Silvestre, 2012, p. 124). Outro fator justificativo, pode estar relacionado com o fator tempo disponível para esta atividade. Poissant et al. (2005) referem no seu artigo que o uso de terminais de cabeceira poupou tempo às enfermeiras, do seu tempo total gasto nos registos durante um turno. Sobre esta questão do tempo, e da gestão eficaz do mesmo, estes autores referem ainda que, em comparação, o uso de desktops ou de terminais centrais eram menos eficientes, aumentando o tempo de trabalho das enfermeiras (de 98,1\% para 328,6\%) por turno. A eficiência da gestão do tempo é um dos muitos benefícios aclamados pelos defensores e implementadores de Registos de Saúde Eletrónicos, no entanto este risco de ineficiência também pode ser uma barreira importante para a uma implementação bem-sucedida (Poissant et al., 2005) e pode explicar algumas evidências encontrada nos dados desta unidade de cuidados, analisada neste trabalho. Muitos conceitos relevantes tem vindo a ser introduzidos, para descrever os cuidados de saúde futuros, alimentados por tecnologias inovadoras de informação e comunicação emergentes, 
vistos como expressões alternativas como o Health-Internet-of-Things (IOT) (Pang, 2013). De facto, a Internet-of-Things promete oferecer soluções promissoras, nomeadamente na área da vigilância de saúde, que em contexto de cuidados hospitalares serão de grande eficácia e eficiência, ao permitir por exemplo monitorizações através de wearable biomedical devices (Pang, 2013) poupando assim tempo e recursos.

A implementação de ferramentas inovadoras deve ter em conta diversos fatores, entre os quais o gasto de tempo na sua utilização, fator que no caso dos enfermeiros pode ser crítico face à escassez de recursos e volume de cuidados, frequentemente complexos. Ao introduzir inovações nos cuidados de saúde, é importante conhecer melhor as determinantes que podem facilitar ou impedir a sua introdução, a fim de conceber uma estratégia adequada para a introdução da inovação (Fleuren et al., 2004). Outra questão que importa abordar, é a maturidade de implementação dos Registos de Saúde Eletrónicos, uma inovação nesta equipa e unidade de cuidados. Os dados obtidos diziam respeito aos dois primeiros anos (2014 e 2015) de implementação do projeto de informatização dos registos, o que poderemos classificar numa fase de iniciação de projeto. Segundo Monteiro (2010) as fases propostas, a propósito da maturidade de que falamos, são enquadradas em dois períodos. O primeiro diz respeito à gestão da tecnologia, com início da integração das tecnologias na organização, dando resposta às necessidades básicas (Monteiro, 2010). Num segundo período ou fase, inicia-se a gestão do recurso informação, começando a haver uma visibilidade do valor da informação na organização, com uma maturidade que permite o planeamento dos sistemas em alinhamento estratégico com a organização, de forma a promover cuidados de saúde de elevada qualidade (Monteiro, 2010). Análises como a realizada neste trabalho permitem um salto qualitativo no processo, permitindo estabelecer planos de ação e correção de práticas, evoluindo assim para planos de maturidade mais avançados, e consequentemente cuidados de saúde de qualidade e sustentáveis.

\section{Conclusões}

A literatura refere diferentes mudanças na Administração Pública, com expressão de diferentes modelos organizacionais. O sector público da saúde têm sido alvo de transformações que diferentes teorias enquadram. A eficácia, a eficiência e a competitividade do New Public Management, constituem alguns dos valores presentes neste sector, sendo cada vez mais importante a utilização de indicadores, objetivos e estratégias de monitorização, e acompanhamento dos mesmos. Outros modelos partilham princípios como a eficiência, como é o caso da Governança Pública (Secchi, 2009). A Governança Pública é considerado um modelo de modernização das organizações públicas, inspirado nos conhecimen- tos do sector privado (Secchi, 2009), que associa mudanças como as verificadas na área da saúde.

Ao analisar os dados presentes na informação padronizada, produzida pelo maior grupo profissional, de uma instituição hospitalar, procuramos responder a algumas questões. Assim, o objetivo geral deste trabalho foi analisar os Registos de Saúde Eletrónicos, pertencente aos utentes, com episódio de internamento numa unidade de cuidados de um hospital público em Portugal, no período de 2014 e 2015. A análise dos dados do Registo de Saúde Eletrónico, produzidos pelos enfermeiros, revelaram potencial para a criação de indicadores e para uma análise da qualidade. Um dos indicadores conseguidos foram as taxas de frequência relativa, sendo possível com estes dados, no entanto, outros como as taxas de incidência e as taxas de prevalência (OE, 2007). Os restantes indicadores, propostos pela Ordem dos Enfermeiros (2007), referentes às taxa de efetividade diagnóstica do risco, taxa de efetividade na prevenção de complicações, Modificações positivas no estado dos diagnósticos, taxas de ganhos possíveis / esperados de efetividade, não foram analisados neste trabalho por impossibilidade de obtenção de alguns dados em tempo útil, uma limitação desta análise, mas um desafio a propor para futuros estudos.

A criação destes indicadores tiveram em atenção a mensurabilidade, através de dados diários do Registo de Saúde Eletrónico, e foram enquadrados nos padrões de qualidade enunciados pela Ordem dos Enfermeiros. Para Silvestre (2012) "(...) os registos de enfermagem assumem um importante papel na definição de indicadores de qualidade dos cuidados de enfermagem. É necessário que os enfermeiros documentem os cuidados que prestam, os diagnósticos, as intervenções e os resultados para que seja possivel evidenciar a qualidade (...)" (p. 121).

Tendo em conta a robustez dos dados analisados, algumas conclusões importantes refletirão impactos ao nível da gestão numa unidade de cuidados, como no caso apresentado. Análises idênticas, nas restantes unidades de cuidados de uma instituição hospitalar, permitiriam a elaboração de conclusões e planos de ação de melhoria global. As principais áreas de melhoria, face aos resultados, poderiam ser: A criação de indicadores de complexidade de cuidados, para apoio à decisão de dotação de recursos e realização de benchmarking; Implementação de inovadores sistemas de gestão da informação, baseados nas novas tecnologias Internet-of-things, com capacidade de conetividade global ao Registo de Saúde Eletrónico (para monitorização da atividade diária através de dispositivos wearable biomedical devices) para registo de informação de forma contínua, poupando recursos e salvaguardando dados importantes (Pang, 2013); Gestão e promoção do desenvolvimento de competências nas áreas com menos expressão, mas importantes para a missão da instituição; Articulação com o sector de saúde ocupacional e 
elaboração de plano de prevenção de complicações e riscos profissionais, associados a atividades e tarefas marcadamente instrumentais, com riscos de lesões causadoras de aumento de absentismo; Implementação de sistemas de vigilância e registos mais próximos dos utentes, para diminuir o tempo gasto pelos enfermeiros (ou outros profissionais) nos registos, aumentando assim a sua eficácia; Divulgação dos resultados na instituição, para promoção da qualidade dos registos, dando evidência aos principais resultados.

O Registo de Saúde Eletrónico permite não apenas a medição de resultados, mas a identificação das melhores práticas clínicas associadas a esses resultados, e deve ser visto como uma ferramenta de gestão e de investigação, que pode apoiar processos de trabalho e de inovação na prestação de cuidados. Outras sugestões para a investigação incluem analisar e avaliar mais indicadores, de forma sistematizada, promover o benchmarking, analisar a qualidade dos dados das notas de alta realizadas e submetidas à Plataforma de Dados da Saúde, em especial pela importância que estas assumem nos Registos de Saúde Eletrónicos e na continuidade dos cuidados ao cidadão.

Nos antigos modelos organizacionais burocráticos considerava-se que a forma de alcançar a eficiência era a hierarquia e a normalização (Peci et al., 2008). Com o aparecimento de novos modelos de gestão, do New Public Management e da Governança, surgiram no discurso e vocabulário da gestão pública novos termos, diferentes necessidades e princípios. O New Public Management apresentou como pontos centrais, os conhecimentos do sector privado para o sector público, com o propósito de diminuir a maquina administrativa, dando ênfase à competição e aumento da eficiência (Peci et al., 2008)

Peci et al. (2008) referem que "(..) a governança, enquanto um novo modelo de gestão pública, requer a conceção de estruturas e processos próprios, que se diferenciam, se é que isto é possivel, de conceções tradicionais da gestão pública." (p. 52). O modelo do New Public Management dá uma ênfase à competição, para o aumento da eficiência, da mesma forma a Governança procura uma eficiência adaptativa, com flexibilidade, experimentação e aprendizagem (Peci et al., 2008). Considerando que, as Tecnologias de Informação e Comunicação podem ser facilitadoras, estes autores apresentam o e-governo como uma ferramenta típica da Governança, ligada à transparência, mas também à eficiência orientada para o cidadão. À semelhança, a informatização do sector da saúde, a análise dos dados referentes aos cuidados, com a extração de indicadores, num processo de melhoria continua, na procura de uma maior eficiência e transparência, enquadra-se em modelos organizacionais baseados nas teorias do New Public Management e Governança, corroborando a posição de autores (Secchi, 2009) de que, no sector público ainda convivem diferentes modelos organizacionais.

\section{Referências bibliográficas}

Bilhim J. Qualificação e Valorização de Competências. Lisboa: Sociedade Portuguesa de Inovação. Coleção Inovação e Governação nas Autarquias; 2004.

Boston Consulting Group [BCG]. Um novo Modelo de Acesso à Inovação em Saúde Baseado em Resultados. Lisboa: Janssen. 2016. Disponível em: https://www.sns.gov.pt.

Carrasqueiro S, Monteiro MH. E-Health Strategic Planning: Defining the E-Health Services Portfolio. Portugal: IGI Global. Portfolio Introduction; 2010.

Correia PM, Jesus 10. Combate às Transferências Bancárias Ilegítimas pela Internet no Direito Português: Entre as Experiências Domésticas e Políticas Globais Concertadas. 2016;12(2):42-563.

Druke H, Klinger P. Networked Public Administration for Better Service: New Production Model for Local Service Delivery in Germany. Innovations in Public Governance. 2011;15(6):140-57. Disponível em: https://www. researchgate.net.

Fleuren M, Wiefferink K, Paulussen T. Determinants of innovation within health care organizations Literature review and Delphi study. International Journal for Quality in Health Care. 2004;16(2):107-23. Disponível em: http://intqhc.oxfordjournals.org.

Harfouche AP. Políticas de Saúde Fundamentação, prioridades, Opções e Resultados. Lisboa: Instituto Superior de Ciências Sociais e Políticas; 2016.

Kierkegaard P, Kaushal R, Vest JR. How could health information ex-change better Meet the needs of care practitioners. Applied Clinical Informatics. 2014;5:861-877. Disponível em: https://www.ncbi.n/m.nih.gov.

Monteiro MH. A adopção de eHealth nos hospitais Públicos em Portugal 1996-2007 [Tese Doutoramento Administração Pública]. Instituto Superior de Ciências Sociais e Políticas; 2010.

National Information Board. Personalised Health and Care 2020 Using Data and Technology to Transform Outcomes for Patients and Citizens. A Framework for Action. UK: NHS. 2014. Disponivel em: https://www.gov.uk.

Odekunle FF. Current Roles and Applications of Electronic Health Record in the Healthcare System. International Journal of Medical Research \& Health Sciences. 2016;5(12):48-51. Disponível em: http://www.jjmrhs.com.

Oncolink [site na Internet]. Portugal: Registo de Saúde Eletrónico. Documento de "Estado da Arte". Administração Central do Sistema de Saúde [ACSS]. Lisboa: Ministério da Saúde. [atualizado 2009; citado 2017]. Disponível em: http://ser.cies.iscte.pt.

Oncolink [site na Internet]. Portugal: Comissão Europeia Comunicação da Comissão ao Parlamento Europeu, ao Conselho, ao Comité Económico e Social Europeu e ao Comité das Regiões. Plano de ação para a saúde em linha, 2012-2020 - Cuidados de saúde inovadores para o século XXI. Bruxelas: Comissão Europeia. [atualizado 2012; citado jan 2017]. Disponível em: http://ec.europa.eu/health//sites/health/files/ehealth/ docs/com_2012_736_pt.pdf.

Oncolink [site na Internet]. World Health Organization [WHO]. World health Statistics. [atualizado 2015; citado 2017]. Disponível em: http://www. who.int/en/.

Oncolink [site na Internet]. Portugal: Ciências da Informação e da Decisão Em Saúde [CIDES] Hospital: Definição e classificação; [atualizado 2016; citado jan 2017]. Disponível em: http://cides.med.up.pt.

Oncolink [site na Internet]. Organization for Economic Cooperation and Development/European Union [OECD/ EU]. Health at a Glance: Europe 2016-State of health in the EU Cycle. Paris: European Commission. [atualizado 2016; citado 2017]. Disponível em: https://www.oecd.org.

Oncolink [site na Internet]. Portugal: Instituto Nacional de Estatística [INE]: Estatísticas da Saúde 2014 Estatísticas Oficiais. [atualizado 2016; citado 2017]. Disponível em: www.ine.pt. 
Oncolink [site na Internet]. Portugal: Organização Mundial de Saúde [OMS]. Conceito de Saúde segundo OMS/WHO.[atualizado 2016; citado 2017]. Disponível em: http://cemi.com.pt/2016/03/04/conceito-de-saudesegundo-oms-who/.

Ordem dos Enfermeiros [OE]. Padrões de Qualidade dos Cuidados de Enfermagem. Enquadramento Conceptual. Enunciados Descritivos. Lisboa: Conselho de Enfermagem; 2001.

Ordem dos Enfermeiros [OE]. Sistema de Informação de Enfermagem (SIE) e Resumo Mínimo de Dados e Core de Indicadores de Enfermagem para o Repositório Central de Dados da Saúde. Lisboa: Ordem dos Enfermeiros; 2007.

Ordem dos Enfermeiros [OE]. Regulamento do Perfil de competências do Enfermeiro de cuidados gerais. Lisboa: Ordem dos Enfermeiros; 2012.

Ordem dos Enfermeiros [OE] \& Conselho Internacional de Enfermeiros [CIE]. Classificação Internacional para a prática de enfermagem [CIPE]. Genebra: Conselho Internacional de Enfermeiros. 2016.

Osbone SP, Radnor Z, Nasi G. A New Theory for Public Management? Toward a (Public) Service Dominant Approach. American Review of Public Administration. 2013;43(2):135-58. Disponível em: http://arp.sagepub. com/content/43/2/135.refs.html.

Peci A, Pieranti OP, Rodrigues S. Governança e New Public Management: Convergências e Contradições no contexto Brasileiro. Revista O\&S. 2008;15(46):39-55. Disponível em: http://www.scielo.br/pdf/osoc/ v15n46/02.pdf.

Pang Z. Technologies and Architectures of the Internet-of-Things (loT) for Health and Well-being. [Tese de Doutoramento]. Sweden: Royal Institute of Technology. 2013. Disponível em: https://pdfs.semanticscholar.org.

Poissant L, Pereira J, Tamblyn R, Kawasumi Y. The impact of electronic health records on time efficiency of physicians and nurses: a systematic review. Journal of the American Medical Informatics Association. 2005;12(5):505516.Disponível em: https://www.ncbi.nlm.nih.gov.
Pontes AC, Leitão IMTA, Ramos IC. Comunicação Terapêutica em Enfermagem: Instrumento essencial do cuidado. Revista Brasileira de Enfermagem REBEn. 2007;61(3):312-8. Disponível em: http://www.scielo. br/pdf/reben/v61n3/a06v61n3.pdf.

Secchi L. Modelos Organizacionais e Reformas da Administração Pública. Revista de Administração Pública. 2009;43(2):347-69. Disponível em: http://www.scielo.br.

Serrano M, Costa A, Costa N. Nursing care: how to develop the competence(ies). Revista de Enfermagem Referência, serlll. 2011;3:15-23. Disponível em: http://www.scielo.mec.pt.

Silva PR. Teoria das Organizações e os Modelos Organizacionais. 2004; Disponível em: https://www.academia.edu.

Silvestre MCC. Os Registos de Enfermagem: um olhar sobre o estado real da saúde das pessoas?. [Tese de Mestrado]. Portugal: Escola Superior de Enfermagem de Coimbra. 2012;1-136. Disponível em: http://repositorio. esenfc.pt.

Simione AA. A modernização da gestão e a governança no setor público em Moçambique. Revista de Administração Pública. 2014;48(3):551-70. Disponível em: http://www.scielo.br.

Simões CMAR, Simões JFFL. Avaliação inicial de Enfermagem em linguagem CIPE segundo as necessidades humanas fundamentais. Revista Referencia-revista de enfermagem. 2007;ll:4:9-23. Disponível em: https:// web.esenfc.pt/.

Taylor N, Williams RC, Hogden E, Braithwaite J, Groene O. Hight performing hospitals: a qualitative systematic review of associated factors and practical strategies for improvement. BMC Health Services Research. BioMed Central. 2015;1-22. Disponível em: https://bmchealthservres. biomedcentral.com

Villax P [Ed.]. Aproveitar o conhecimento, a tecnologia e a inovação. Grupo de trabalho 4. Um futuro para a saúde. Lisboa: Fundação Calouste Gulbenkian. 2015. Disponível em: https://gulbenkian.pt. 